\title{
Validation of the Persian Smartphone Addiction Scale Among Tehran University Students, Iran
}

\author{
Ali Kheradmand (iD) ${ }^{1}$, Elham Sadat Amirlatifi (ii) ${ }^{2,{ }^{*}}$, Mohammad-Reza Sohrabi (ii ${ }^{3}$ and Azadeh Mazaheri \\ Meybodi $^{1}$ \\ ${ }^{1}$ Taleghani Hospital Research Development Committee, Faculty of Medicine, Shahid Beheshti University of Medical Sciences, Tehran, IR Iran \\ ${ }^{2}$ School of Medicine, Shahid Beheshti University of Medical Sciences, Tehran, IR Iran \\ ${ }^{3}$ Department of Community Medicine, Shahid Beheshti University of Medical Sciences, Tehran, IR Iran \\ "Corresponding author: School of Medicine, Shahid Beheshti University of Medical Sciences, Tehran, IR Iran. Email: e_amirlatifi@yahoo.com
}

Received 2018 June 23; Revised 2019 March 06; Accepted 2019 March 09.

\begin{abstract}
Background: Smartphone addiction is a recent phenomenon which has been increasing tremendously as new applications are being published, especially in Southeast Asia. In Iran, smartphone users are estimated to be around 46 million people and are increasing day by day.

Objectives: This study was conducted to evaluate the psychometric properties of persian smartphone addiction scale (PSAS) among students of Tehran universities, Iran.

Patients and Methods: This cross-sectional study was conducted in 2017. The PSAS and Internet addiction test (IAT), which has been already validated in Iran, were completed by 200 students studying at Tehran universities who were selected by the multistage random sampling method

Results: A comparison was made between SAS and IAT in order to find out the concurrent validity. Pearson's correlation coefficient was $0.7(\mathrm{P}=0.00)$, showing a strong positive correlation between PSAS and Persian IAT. The optimal cutoff score was more than 106 for at-risk individuals by analyzing the receiver operating characteristic (ROC) curve. The sensitivity and specificity of the test were $80 \%$ and $86 \%$, respectively, and the Cronbach's alpha coefficient was 0.93 for the total scale. Test-retest reliability of PSAS was high, with an interclass correlation of 0.996. Sample adequacy for factor analysis as represented by Kaiser-Mayer-Olkin (0.92) and Bartlett's test of sphericity was significant $(\mathrm{P}=0.00)$, indicating that factor analysis was useful.
\end{abstract}

Conclusions: PSAS is a valid and reliable tool for evaluating smartphone addiction in the Iranian population.

Keywords: Smart Phone, Behavior, Addictive, Validation Studies, University, Psychometrics

\section{Background}

With numerous applications, smartphones provide their users web-based communication, business, education, entertainment, and clinical utilization. Given their assortment of capacities and usability, the number worldwide users was in excess of 1.08 billion in 2012, and it is expanding at a rapid rate. However, smartphones can be harmful when used excessively (1). For instance, uncontrolled smartphone utilization can cause maladaptive behavioral difficulties found in impulse control disorders or pathological gambling; it can impede school or work, diminish academic ability, decrease real-life social interaction and result in relationship issues. It can also lead to health problems such as blurred vision and pain in the wrists or neck $(2,3)$.

Addiction is a phenomenon described by tolerance, withdrawal, dependence, and social problems, and researchers have proposed the idea of "smartphone addiction". In spite of the fact that smartphone addiction is generally a new idea and has not been mentioned in the latest version of the diagnostic and statistical manual for mental disorders (DSM-V), investigations have been performed to address the adverse effects of cellphone dependence. Furthermore, DSM-V has confirmed the concept of behavioral addiction through gambling disorder in the "substancerelated and addictive behavior" category and accepted the research criteria for game addiction (4).

In Iran, there are no official reports regarding the number of smartphone users, but it is estimated that there are at least 40 million smartphones in the country (5). Recent data also showed that there are some 40 million active social media users, constituting almost half of the population (6). 
However, the issue of smartphone addiction has not been studied so far in Iran. Since this problem is prominent in the youth, especially in university students, it can cause numerous problems in occupational and academic performances and interpersonal relationships; thus, it seems necessary to investigate smartphone dependency in this population $(7,8)$. To this end, a scale should be validated to evaluate smartphone addiction among the Iranian population.

The first scale for smartphone dependency was created by Kwon et al. and includes 33 items and has been stated to be reliable, with good internal consistency (Cronbach's alpha $=0.967)$. The concurrent validity of the six subscales of this tool was appropriate (9).

Since smartphone addiction has similar characteristics to Internet addiction, Internet addiction test (IAT) can be used to develop a smartphone addiction test. First, smartphone addiction scale (SAS) was created base on the K-scale, a 40-item scale for identifying Internet addiction among the Korean juvenile population. Then, 10 items on the specific characteristics of smartphones were added. After final analysis, the SAS consisting of 33 items was prepared (9).

\section{Objectives}

The aim of this study was to evaluate the psychometric properties of the Persian version of the SAS (PSAS), which could help future studies on smartphone addiction in the Iranian population.

\section{Patients and Methods}

\subsection{Study Design}

We performed this cross-sectional study at Shahid Beheshti Medical University (SBMU) in 2017. Initially, the English version of SAS (9) was translated into Persian by a bilingual translator, and then it was back translated to English by another translator, in order to establish the accuracy of the translation. Therefore, all mismatches between the original version and the back-translated were one rectified. This was how the first revision of the Persian SAS was prepared.

Afterwards, the first revision was distributed among 20 medical university students as pilot volunteers to examine its face validity. Then, inappropriate phrases and terms were eliminated and replaced according to the participants' comments, which resulted in the final revision of the Persian SAS questionnaire.

The final revision of the PSAS was studied by a group of 20 psychiatrists and a Professor of Community Medicine, who were familiar with psychometric tools, to evaluate the sufficiency of semantic equivalence.

Some slight changes were made to some items; for instance, social networks like Twitter and Facebook were replaced with Telegram and Instagram due to their higher popularity in Iran. However, no items were eliminated or added.

The PSAS and IAT, which has been already validated in Iran (10), were completed by 200 students from Tehran universities. The sociodemographic variables (i.e., age, gender, major, and educational degree), were collected by a researcher-made checklist. Meanwhile, information regarding the usage of mobile Internet, social networks, and game applications was gathered.

\subsection{Participants}

A total of 200 university students from different faculties in Tehran were selected with the multistage cluster sampling method. A written informed consent was obtained from all the participants once we explained the research objectives to them. The study was approved by the Ethics Committee of Shahid Beheshti Medical University (2017,309).

\subsection{Instruments}

\subsubsection{Smartphone Addiction Scale}

SAS is a scale for smartphone addiction that comprises six factors and 33 items, rated using a six-point Likert scale (i.e., from 1: strongly disagree to 6: strongly agree) primarily based on self-reporting. The six factors are positive expectation, daily-life disturbance, Internet-based relationship, overuse, withdrawal, and tolerance. The six subscales' scores are summed up to yield a total SAS score. The minimum and maximum SAS scores ranges from 48 to 288 , with higher scores suggesting more severe dependency. The Cronbach's alpha for the total scale was 0.967. The correlation between the subscales of SAS and Young scale (Y-scale) was estimated and the results showed that all the subscales were positively related to those of Y-scale $(9,11)$.

\subsubsection{Internet Addiction Test}

IAT is the most popular instrument for diagnosing Internet addiction, which was initially created by Young in 1998 (9). the Persian version of Young's IAT has perfect psychometric properties and is a valid and reliable tool for psychological and psychiatric studies in order to distinguish normal Internet users from Internet addicts. In previous investigations, content and convergent validity, internal consistency $(\alpha=0.88)$ and test-retest reliability $(\mathrm{r}=0.82)$ have been determined and all were acceptable. The best cutoff point for the questionnaire was 46 (10). 


\subsection{Statistical Analysis}

All the analyses were conducted by SPSS version 22.0. Descriptive statistics were used for sociodemographic characteristics of the participants. Concurrent validity between PSAS and the Persian IAT was established by Pearson's correlation. Basically, the ideal SAS cutoff score for at-risk cases was determined by coordinate points, whilst the score for the Persian version of IAT was greater than 46 (10). At this cutoff point, based on ROC analyses, specificity and sensitivity were at an optimal level. Internal consistency was assessed by Cronbach's alpha, and test-retest reliability was confirming for the external consistency of the scale. Principal component analysis and varimax with Kaiser normalization were used for establishing construct validity. Factor loading of more than 0.30 and eigenvalue greater than 1 were considered for factor analysis (12).

\section{Results}

Out of 200 participants, 124 (62\%) were female. The majority of the students were single and aged between 20 and 29 years and had high levels of education. Also, 97.5\% of the respondents had access to the Internet on their smartphones. What's more, 98\% used social network services, and 55\% used game applications. Table 1 shows the sociodemographic characteristics of the participants, the mean score of SAS in our study was 94 with the standard error of 26.97 ( $\min$ score $=36$, $\max$ score $=158$ ).

\subsection{Concurrent Validity}

A comparison was made between SAS and IAT in order to find out the concurrent validity of the PSAS. Pearson's correlation coefficient was $0.7(\mathrm{P}=0.00)$, showing a significant positive correlation between PSAS and Persian IAT.

\subsection{Cutoff Point, Specificity, and Sensitivity}

To determine the sensitivity and specificity of SAS, information was analyzed by ROC curve in SPSS software. The area under the curve (AUC) for the ROC curve was calculated at $0.915(95 \% \mathrm{CI}=0.869-0.962)$. The ideal cutoff score for recognizing smartphone dependent patient was more than 106 , with a high sensitivity (80\%) and specificity (86\%). (Figure 1 and Table 2) Based on a score of 106, the prevalence of at-risk persons for smartphone addiction was 32\% in our study.

\subsection{Internal Consistency}

Cronbach's alpha coefficient for the whole instrument was 0.93 , and the separate coefficients for the subscales of daily-life disturbance, positive expectation, withdrawal, Internet-based relationship, overuse, and tolerance were

\begin{tabular}{|c|c|}
\hline Variable & No. $(\%)$ \\
\hline \multicolumn{2}{|l|}{ Gender } \\
\hline Female & $124(62)$ \\
\hline Male & $76(38)$ \\
\hline \multicolumn{2}{|l|}{ Age } \\
\hline$<20$ & $12(6)$ \\
\hline $20-29$ & $136(68)$ \\
\hline $30-39$ & $35(17.5)$ \\
\hline$>40$ & $17(8.5)$ \\
\hline \multicolumn{2}{|l|}{ Marital status } \\
\hline Single & $154(77)$ \\
\hline Married & $46(23)$ \\
\hline \multicolumn{2}{|l|}{ Major } \\
\hline Medical sciences & $136(68)$ \\
\hline Engineering & $10(5)$ \\
\hline Basic sciences & $6(3)$ \\
\hline Humanities and social sciences & $48(24)$ \\
\hline \multicolumn{2}{|l|}{ Educational degree } \\
\hline Advanced diploma & $10(5)$ \\
\hline Bachelor's & $30(15)$ \\
\hline Master's & $30(15)$ \\
\hline Doctorate & $130(65)$ \\
\hline \multicolumn{2}{|l|}{ Mobile Internet } \\
\hline Yes & $195(97.5)$ \\
\hline No & $5(2.5)$ \\
\hline \multicolumn{2}{|l|}{ Social network services } \\
\hline Yes & $196(98)$ \\
\hline No & $4(2)$ \\
\hline \multicolumn{2}{|l|}{ Game applications } \\
\hline Yes & $110(55)$ \\
\hline No & $90(45)$ \\
\hline
\end{tabular}

$0.817,0.876,0.833,0.806,0.749$ and 0.767 , respectively. The alpha coefficient would have changed between 0.936 and 0.940 if any items were deleted (Table 3).

\subsection{External Consistency}

PSAS was completed again after a two-week interval by 70 the study samples. Comparison of mean scores was made by paired $t$-test (Table 4 ), yielding a correlation coefficient of 0.997 which shows no significant differences $(\mathrm{P}=$ 0.394). This indicates that the test-retest reliability of the PSAS is high. Interclass correlation was 0.996 (95\% confidence interval $=0.994-0.998$ ). 


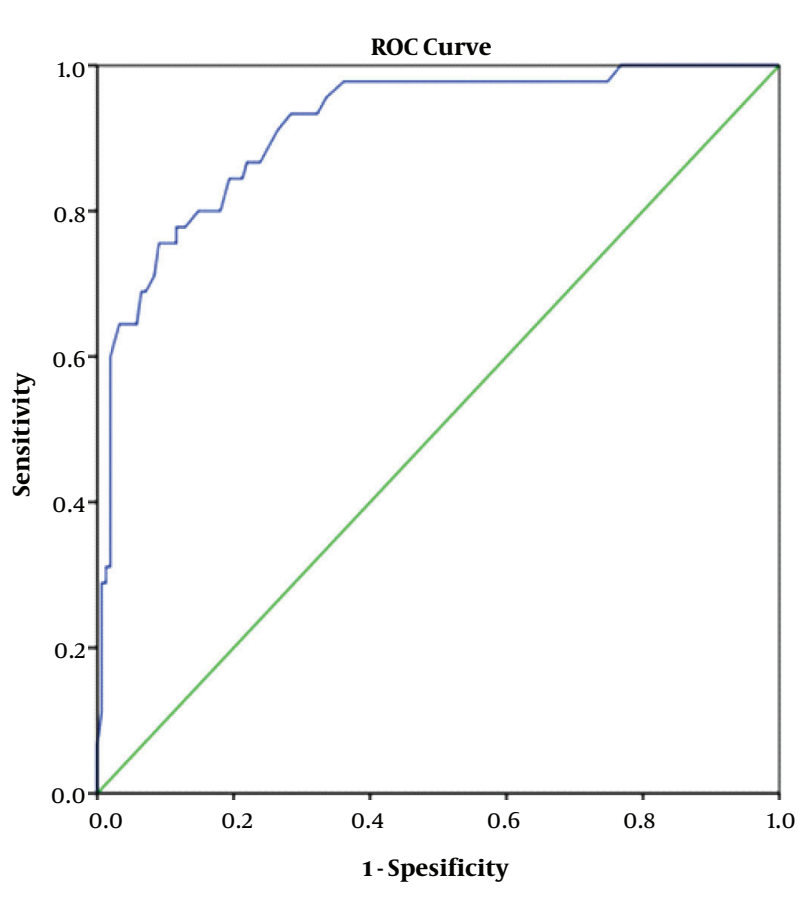

Figure 1. Receiver operating characteristic curve

\subsection{Factor Analysis}

Sample adequacy for factor analysis as represented by Kaiser-Mayer-Olkin (0.92) and Bartlett's test of sphericity was significant $(P=0.00)$, indicating factor analysis was useful. Six factors were extracted (Eigenvalue $>1.00$ ) via principal component analysis. Varimax rotation with Kaiser normalization explained $62.85 \%$ of the total variance (Table 5) This result was approximately compatible with those of the original SAS (9).

\section{Discussion}

This study was the first attempt to evaluate the psychometric properties of the PSAS. Our results demonstrated that PSAS is a valid and reliable tool for evaluating smartphone dependency in the Iranian population.

This study exhibited that internal consistency, dimensionality, and concurrent and construct validity of the PSAS were desirable. Pearson's correlation coefficient indicated a strong positive correlation between PSAS and IAT. The results of Ching et al. study in the Malay population indicated that all SAS subscales, except positive expectation, correlated with the Malay version of the IAT (13). The six dominant components that explained a large proportion of the variability of the SAS-M were similar to those of the

\begin{tabular}{|c|c|c|}
\hline Cutoff & Sensitivity & 1-Specificity \\
\hline 90.50 & 0.978 & 0.426 \\
\hline 91.50 & 0.978 & 0.400 \\
\hline 92.50 & 0.978 & 0.381 \\
\hline 93.50 & 0.978 & 0.361 \\
\hline 94.50 & 0.956 & 0.335 \\
\hline 95.50 & 0.933 & 0.323 \\
\hline 96.50 & 0.933 & 0.303 \\
\hline 97.50 & 0.933 & 0.284 \\
\hline 98.50 & 0.911 & 0.265 \\
\hline 99.50 & 0.867 & 0.239 \\
\hline 100.50 & 0.867 & 0.219 \\
\hline 101.50 & 0.844 & 0.213 \\
\hline 102.50 & 0.844 & 0.206 \\
\hline 103.50 & 0.844 & 0.194 \\
\hline 104.50 & 0.800 & 0.181 \\
\hline 105.50 & 0.800 & 0.161 \\
\hline 106.50 & 0.800 & 0.148 \\
\hline 107.50 & 0.778 & 0.129 \\
\hline 108.50 & 0.778 & 0.116 \\
\hline 109.50 & 0.756 & 0.116 \\
\hline 110.50 & 0.756 & 0.090 \\
\hline 111.50 & 0.711 & 0.084 \\
\hline 112.50 & 0.689 & 0.071 \\
\hline 113.50 & 0.689 & 0.065 \\
\hline 114.50 & 0.644 & 0.058 \\
\hline 115.50 & 0.644 & 0.039 \\
\hline 116.50 & 0.644 & 0.032 \\
\hline 118.50 & 0.622 & 0.026 \\
\hline 122.00 & 0.600 & 0.019 \\
\hline
\end{tabular}

original SAS. In the present study and original SAS, the components consisted of daily-life disturbance, positive expectation, withdrawal, Internet-based relationship, overuse, and tolerance. The factors of the Persian scale were not parallel to original scale in factor analysis. This can be the result of the differences between the Iranian and Korean samples.

All the PSAS subscales were significantly related to the Persian version of the IAT, showing that PSAS had good concurrent validity.

Although our study population were homogenous as all subjects were students of Tehran Universities with a nar- 


\begin{tabular}{|c|c|c|c|c|}
\hline & Scale Mean if Item Deleted & Scale Variance if Item Deleted & Corrected Item-Total Correlation & Cronbach's Alpha if Item Deleted \\
\hline $\mathbf{q 1}$ & 90.58 & 686.366 & 0.540 & 0.938 \\
\hline $\mathbf{q 2}$ & 90.48 & 689.135 & 0.515 & 0.938 \\
\hline q3 & 91.52 & 695.950 & 0.423 & 0.939 \\
\hline q4 & 91.39 & 698.641 & 0.358 & 0.940 \\
\hline q5 & 91.37 & 689.017 & 0.514 & 0.938 \\
\hline q6 & 90.67 & 694.214 & 0.465 & 0.938 \\
\hline $\mathbf{q 7}$ & 90.83 & 688.232 & 0.543 & 0.938 \\
\hline q8 & 91.44 & 696.157 & 0.479 & 0.938 \\
\hline q9 & 91.34 & 689.942 & 0.547 & 0.938 \\
\hline q10 & 92.07 & 693.945 & 0.557 & 0.938 \\
\hline q11 & 91.78 & 682.585 & 0.611 & 0.937 \\
\hline q12 & 91.78 & 690.585 & 0.558 & 0.938 \\
\hline q13 & 91.90 & 688.995 & 0.603 & 0.937 \\
\hline q14 & 90.55 & 671.124 & 0.667 & 0.936 \\
\hline q15 & 90.89 & 674.711 & 0.728 & 0.936 \\
\hline q16 & 91.46 & 675.908 & 0.709 & 0.936 \\
\hline q17 & 91.59 & 681.610 & 0.621 & 0.937 \\
\hline q18 & 91.49 & 680.663 & 0.621 & 0.937 \\
\hline q19 & 91.94 & 700.499 & 0.348 & 0.940 \\
\hline q20 & 90.48 & 704.904 & 0.300 & 0.940 \\
\hline q21 & 91.91 & 697.610 & 0.471 & 0.938 \\
\hline q22 & 91.70 & 675.206 & 0.673 & 0.936 \\
\hline q23 & 92.15 & 696.098 & 0.519 & 0.938 \\
\hline q24 & 90.81 & 675.183 & 0.620 & 0.937 \\
\hline q25 & 90.04 & 674.265 & 0.668 & 0.936 \\
\hline q26 & 92.27 & 698.960 & 0.532 & 0.938 \\
\hline q27 & 89.99 & 702.568 & 0.322 & 0.940 \\
\hline q28 & 90.37 & 676.886 & 0.525 & 0.938 \\
\hline q29 & 90.40 & 672.774 & 0.657 & 0.936 \\
\hline q30 & 91.13 & 670.224 & 0.735 & 0.936 \\
\hline q31 & 91.38 & 688.055 & 0.557 & 0.938 \\
\hline q32 & 90.17 & 689.535 & 0.453 & 0.939 \\
\hline q33 & 91.02 & 667.412 & 0.692 & 0.936 \\
\hline
\end{tabular}

\begin{tabular}{|c|c|c|c|}
\hline & Mean \pm SD & $\mathbf{N}$ & Mean Standard Error \\
\hline Test & $97.40 \pm 24.862$ & 70 & 2.972 \\
\hline Retest & $97.61 \pm 24.033$ & 70 & 2.873 \\
\hline
\end{tabular}

rower range of occupation and education compared to the

wide range in the original SAS study, all the components were the same as those in the original SAS.

PSAS had good internal consistency; the Cronbach's alpha was 0.93 in this study and the separate coefficients for the daily-life disturbance, positive expectation, withdrawal, Internet-based relationship, overuse, and tolerance subscales of PSAS were $0.817,0.876,0.833,0.806,0.749$ and 0.767 , respectively. Demirci et al. reported a similar 


\begin{tabular}{|c|c|c|c|c|c|c|}
\hline \multirow{2}{*}{ Question No. } & \multicolumn{6}{|c|}{ Components } \\
\hline & Overuse & Withdrawal & Tolerance & Positive Expectation & Cyberspace-Oriented Relationship & Daily Life Disturbance \\
\hline Q1 & & & 0.616 & & & \\
\hline Q2 & & & & & & 0.522 \\
\hline Q3 & & & & & & 0.820 \\
\hline Q4 & & & & & & 0.765 \\
\hline Q5 & & & & & & 0.671 \\
\hline Q6 & & & & 0.755 & & \\
\hline Q7 & & & & 0.799 & & \\
\hline Q8 & & & & 0.716 & & \\
\hline Q9 & & & & 0.756 & & \\
\hline Q10 & & 0.672 & & & & \\
\hline Q11 & & 0.717 & & & & \\
\hline Q12 & & & & 0.498 & & \\
\hline Q13 & & 0.721 & & & & \\
\hline Q14 & & 0.489 & & & & \\
\hline Q15 & & 0.533 & & & & \\
\hline Q16 & & 0.418 & & & & \\
\hline Q17 & & 0.610 & & & & \\
\hline Q18 & 0.448 & & & & & \\
\hline Q19 & & 0.459 & & & & \\
\hline Q20 & & & 0.412 & & & \\
\hline Q21 & & & & & 0.814 & \\
\hline Q22 & & & & & 0.501 & \\
\hline Q23 & & & & & 0.799 & \\
\hline Q24 & 0.487 & & & & & \\
\hline Q25 & 0.748 & & & & & \\
\hline Q26 & & & & & 0.687 & \\
\hline Q27 & 0.627 & & & & & \\
\hline Q28 & 0.637 & & & & & \\
\hline Q29 & 0.526 & & & & & \\
\hline Q30 & 0.518 & & & & & \\
\hline Q31 & & & 0.731 & & & \\
\hline Q32 & & & 0.797 & & & \\
\hline Q33 & & & 0.516 & & & \\
\hline Eigenvalue & 11.702 & 2.868 & 1.942 & 1.664 & 1.342 & 1.225 \\
\hline Variance (\%) & 35.461 & 8.692 & 5.883 & 5.041 & 4.066 & 3.713 \\
\hline Cumulative (\%) & 35.46 & 44.15 & 50.03 & 55.07 & 59.14 & 62.85 \\
\hline
\end{tabular}

${ }^{\text {a }}$ Extraction method: principal component analysis; rotation method: varimax with Kaiser normalization.

finding (Cronbach's alpha coefficient $=0.947$ ) in the validation of the Turkish version of the SAS (14). Also, Ching et al. reported an internal consistency of 0.94 in the Malay version of SAS (13).

Test-retest reliability of the PSAS was high and its interclass correlation was 0.996 , which are even better than those of the original version of the SAS and the Malay version of SAS with the interclass correlations of 0.95 (13).

The PSAS had good external consistency. Lin et al. examined the test-retest reliability of SAS among 85 participants, which yielded intraclass correlations of $0.80-0.91$ $(\mathrm{P}<0.001)(14)$.

In this study, ROC analysis determined the score of 106 as the cutoff point, with a test sensitivity of $80 \%$ and specificity of $86 \%$. In the Malay population, the optimal cutoff point for smartphone addiction was 98 , while the score for the Malay version of the IAT was more than 43. The prevalence of smartphone addiction in the Malay population was $46.9 \%$ based on this cutoff score (13). The difference in outcomes may be due to demographic diversities in the two populations. However, in the present study, considering addiction as a stigma, attempts were made for increasing the specificity of the test to minimize falsepositive cases, so a higher score was achieved as the cutoff point.

The prevalence of Internet addiction was $22.5 \%$ in our 
study. This result is similar to the findings of Modara et al. in 2017 in Iran. They showed that Internet addiction rate had increased from 2006 to 2015 in Iran (15).

The prevalence of smartphone addiction was $32 \%$. This percentage varies in studies of different populations. In a survey by Lee, about $11 \%$ of African-American college students demonstrated an abnormal state of smartphone addiction and 10\% scored a high level of Facebook addiction (16). In Switzerland, smartphone addiction was found in $16.9 \%$ of students (17). The prevalence of problematic mobile phone use in British adolescents was 10\% (18). Also, it seems that smartphone addiction is highly frequent among students in Tehran universities.

In Asian countries, the overall prevalence of smartphone ownership is 62\% (19) and smartphone addiction rate is estimated higher than Iran. In Cha and Seo study, $30.9 \%$ of middle school students in Korea were classified as a high-risk group for smartphone addiction (20).

Considering the above-mentioned statistics, it seems that the prevalence of smartphone addiction among students is high. Thus, it is necessary to perform further investigations for identifying high-risk groups and taking some measures to control this phenomenon.

\subsection{Limitations}

Despite the adequacy of the sample size, the study was conducted among university students without any considerable functional impairments, which makes it difficult to generalize the findings to clinical conditions.

It was not possible to use clinical interviews for the diagnosis of smartphone addiction in this study. Because there are no established criteria for smartphone addiction as indicated by DSM V. However, our findings could be used by future studies to develop other clinical tools for the diagnosis of smartphone addiction.

\section{Acknowledgments}

This research was supported by Taleghani Hospital Research Development Committee at Shahid Beheshti University of Medical Sciences, Shiraz, IR Iran. We appreciate the cooperation of the supporters and participants.

\section{Footnotes}

Authors' Contribution: All authors (Ali Kheradmand, Elham Sadat Amirlatifi, Mohammad-Reza Sohrabi and Azadeh Mazaheri Meybodi) participated in the study design, the conception of research questions and drafting of the paper. Ali Kheradmand and Elham Sadat Amirlatifi led the work and drafted the manuscript.
Conflict of Interests: The authors declare that they have no competing interests.

Ethical Approval: The study was approved by the Ethics Committee of Shahid Beheshti Medical University $(1395,309)$. The study was conducted based on Helsinki Declaration.

Funding/Support: This research was supported by Taleghani Hospital Research Development Committee at Shahid Beheshti University of Medical Sciences, Shiraz, Iran.

Patient Consent: A written informed consent was provided by each participant once they acquired a complete clarification of the study objectives.

\section{References}

1. Sim MS, Kim EM. The smart phone use survey. Seoul: Korea Communications Commission Press; 2011.

2. Mok JY, Choi SW, Kim DJ, Choi JS, Lee J, Ahn H, et al. Latent class analysis on Internet and smartphone addiction in college students. Neuropsychiatr Dis Treat. 2014;10:817-28. doi:10.2147/NDT.S59293. [PubMed: 24899806]. [PubMed Central: PMC4038421].

3. Alejalil N, Davoodi SR. Mobile phone usage and its effects on pedestrians' distraction. Int J High Risk Behav Addict. 2016;6(3). doi: 10.5812/ijhrba.35431.

4. Choi SW, Kim DJ, Choi JS, Ahn H, Choi EJ, Song WY, et al. Comparison of risk and protective factors associated with smartphone addiction and Internet addiction. J Behav Addict. 2015;4(4):308-14. doi: 10.1556/2006.4.2015.043. [PubMed: 26690626]. [PubMed Central: PMC4712765].

5. Ghasemi Naraghi H. [The number of smartphone users in Iran reaches 40 million]. 2018, [cited 2015 May 13]. Persion. Available from: https:// digiato.com/?p=51054.

6. Financial Tribune. [Latest data on iran surge in social media use]. 2019, [cited 2018 Feb 6]. Persian. Available from: https://financialtribune. com/node/81536.

7. Chiu SI. The relationship between life stress and smartphone addiction on taiwanese university student: A mediation model of learning self-efficacy and social self-efficacy. Comput Hum Behav. 2014;34:49-57. doi: 10.1016/j.chb.2014.01.024.

8. Samaha M, Hawi NS. Relationships among smartphone addiction, stress, academic performance, and satisfaction with life. Comput Hum Behav. 2016;57:321-5. doi: 10.1016/j.chb.2015.12.045.

9. Kwon M, Lee JY, Won WY, Park JW, Min JA, Hahn C, et al. Development and validation of a smartphone addiction scale (SAS). Plos One. 2013;8(2).

10. Alavi SS, Eslami M, Maracy MR, Najafi M, Jannatifard F, Rezapour H. Psychometric properties of young Internet addiction test. Int J Behav Sci. 2010;4(3):183-9.

11. Kwon M, Kim DJ, Cho H, Yang S. The smartphone addiction scale: Development and validation of a short version for adolescents. PLoS One. 2013;8(12). e83558. doi: 10.1371/journal.pone.0083558. [PubMed: 24391787]. [PubMed Central: PMC3877074].

12. Institute for Digital Research and Education. A practical introduction to factor analysis: Exploratory factor analysis. UCLA: Statistical consulting group. 2019. Available from: https://stats.idre.ucla.edu/spss/ seminars/introduction-to-factor-analysis/a-practical-introductionto-factor-analysis.

13. Ching SM, Yee A, Ramachandran V, Sazlly Lim SM, Wan Sulaiman WA, Foo YL, et al. Validation of a malay version of the smartphone 
addiction scale among medical students in Malaysia. PLoS One. 2015;10(10). e0139337. doi: 10.1371/journal.pone.0139337. [PubMed: 26431511]. [PubMed Central: PMC4592235].

14. Lin YH, Chang LR, Lee YH, Tseng HW, Kuo TB, Chen SH. Development and validation of the smartphone addiction inventory (SPAI). PLoS One. 2014;9(6). e98312. doi: 10.1371/journal.pone.0098312. [PubMed: 24896252]. [PubMed Central: PMC4045675].

15. Modara F, Rezaee-Nour J, Sayehmiri N, Maleki F, Aghakhani N, Sayehmiri K, et al. Prevalence of Internet addiction in Iran: A systematic review and meta-analysis. Addict Health. 2017;9(4):243-52. [PubMed: 30574288]. [PubMed Central: PMC6294487].

16. Lee EB. Too much information: Heavy smartphone and facebook utilization by African American young adults. J Black Stud. 2014;46(1):4461. doi: $10.1177 / 0021934714557034$.

17. Haug S, Castro RP, Kwon M, Filler A, Kowatsch T, Schaub MP.
Smartphone use and smartphone addiction among young people in Switzerland. J Behav Addict. 2015;4(4):299-307. doi 10.1556/2006.4.2015.037. [PubMed: 26690625]. [PubMed Central: PMC4712764].

18. Lopez-Fernandez O, Honrubia-Serrano L, Freixa-Blanxart M, Gibson W Prevalence of problematic mobile phone use in British adolescents. Cyber Psychol Behav Soc Network. 2014;17(2):91-8.

19. Mak KK, Lai CM, Watanabe H, Kim DI, Bahar N, Ramos M, et al. Epidemiology of Internet behaviors and addiction among adolescents in six Asian countries. Cyberpsychol Behav Soc Netw. 2014;17(11):720-8. doi: 10.1089/cyber.2014.0139. [PubMed: 25405785].

20. Cha SS, Seo BK. Smartphone use and smartphone addiction in middle school students in Korea: Prevalence, social networking service, and game use. Health Psychol Open. 2018;5(1):2.055102918755E+14. doi: $10.1177 / 2055102918755046$. 